\title{
Photon-jet correlations at RHIC and the LHC
}

\author{
Michael Klasen*† \\ Institut für Theoretische Physik, Westfälische Wilhelms-Universität Münster, \\ Wilhelm-Klemm-Straße 9, D-48149 Münster, Germany \\ E-mail: michael.klaseneuni-muenster.de
}

We present POWHEG predictions for photon-jet correlations at RHIC and the LHC. We show that the total transverse-momentum spectrum of photons and jets is modified not only by medium, but also by higher-order QCD effects, as is the distribution in their relative azimuthal angle. At the LHC, photon-jet measurements in the forward region allow to probe parton momentum fractions of lead ions down to $10^{-4}$.

EPS-HEP 2017, European Physical Society conference on High Energy Physics 5-12 July 2017

Venice, Italy

* Speaker.

${ }^{\dagger}$ Work supported by the BMBF under contract $05 \mathrm{H} 15 \mathrm{PMCCA}$. 


\section{Introduction}

Photon-jet correlations are important probes of the hot medium produced in heavy-ion collisions [1]. In particular, the transverse momentum $p_{T}$ of the photon, which does not undergo strong interactions with the Quark-Gluon-Plasma (QGP), allows to calibrate the $p_{T}$ of the jet at the time of its creation. A comparison of the observed jet- $p_{T}$ with the photon- $p_{T}$ then gives a quantitative measure of jet energy loss in the medium. Similarly, the azimuthal angle difference $\Delta \phi$ between the jet and the photon is modified by the QGP, whereas one expects both to be back-to-back in vacuo. This simple picture is, however, based on perturbative QCD at leading order (LO) and, as we will see in this contribution, must be modified in next-to-leading order (NLO) and beyond.

Up to now, photon-jet correlations have been either analysed with LO Monte Carlo generators like PYTHIA [2] or with NLO calculations like JETPHOX [3]. While the former suffer from large theoretical scale uncertainties, the latter have only up to three partons in the final state and thus insufficient detail to describe all of the experimental observables. We have recently combined NLO calculations with parton showers (PS) for photon production using the POWHEG method [4] and applied them to data from RHIC [5] and the LHC [6].

Typical results for photon-jet correlations at BNL's Relativistic Heavy-Ion Collider RHIC are shown in Sec. 2. In Sec. 3, we demonstrate how forward measurements of proton-lead collisions at CERN's Large Hadron Collider LHC can give access to nuclear parton distribution functions (nPDFs) at very low values of the parton momentum fraction $x$. We then summarise our results in Sec. 4.

\section{Photon-jet correlations at RHIC}

The PHENIX collaboration has measured photon-hadron jet correlations in pp collisions at a centre-of-mass energy of $\sqrt{s}=200 \mathrm{GeV}$ [7]. Photons and charged hadrons were detected at central rapidity $\left|\eta^{\gamma, h}\right|<0.35$. In Fig. 1 we show the distribution in the combined photon-hadron tranverse momentum $p_{T}^{\gamma h}$. The non-perturbative region is avoided by applying individual cuts on $p_{T}^{\gamma, h}>1$ $\mathrm{GeV}$. At sufficiently high net $p_{T}^{\gamma h}$, the NLO+PS corrections (red) to the LO prediction (green) are small, since all relevant scales are large. The interesting region used for jet calibration is the region of small $p_{T}^{\gamma h}$. While the individual transverse momenta still allow the application of perturbation theory, higher-order corrections become large due to soft gluon emission and must be resummed to all orders. This is done reliably either with analytic resummation methods or, as is the case here, by combining the NLO calculation with a PS.

To determine the azimuthal correlations, photons and jets were accepted by PHENIX above transverse momenta $p_{T}^{\gamma, h}$ of 5 and $3 \mathrm{GeV}$, respectively. The corresponding results are shown in Fig. 2, subtracted to exhibit Zero Yield at the Minimum (ZYAM). The away-side region with photons and jets in opposite hemispheres $(\Delta \phi \sim \pi)$ is reasonably described already at LO (green). At $\mathrm{NLO}+\mathrm{PS}$ (red), the rise in this region is enhanced and closer to the data. More important is the near-side or fragmentation region, where the LO predictions remain unrealistically flat, while those at NLO+PS follow the rise of the data. 


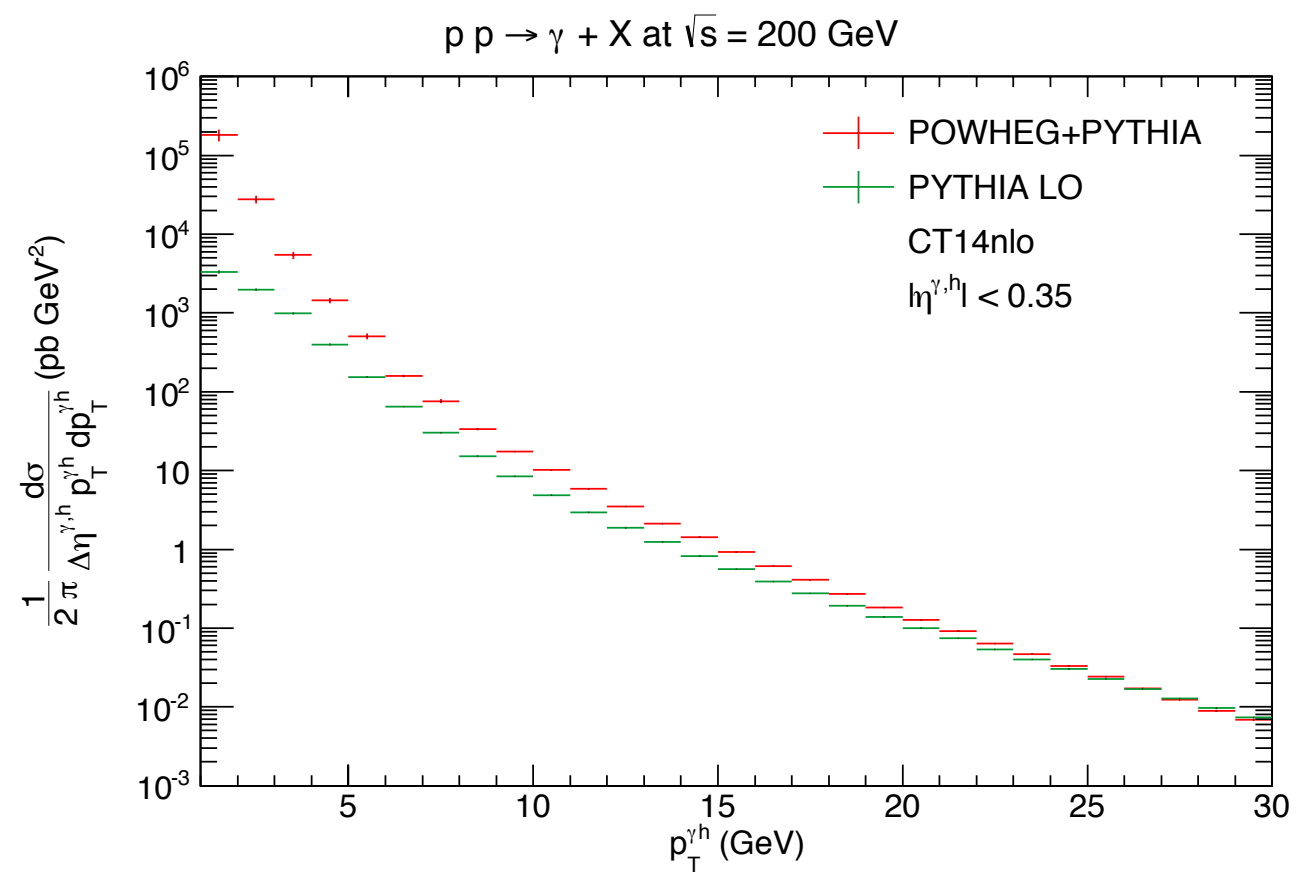

Figure 1: Transverse-momentum spectrum of the photon-hadron pair in pp collisions with $\sqrt{s}=200 \mathrm{GeV}$ at RHIC in LO+PS (green) and NLO+PS (red) [5].

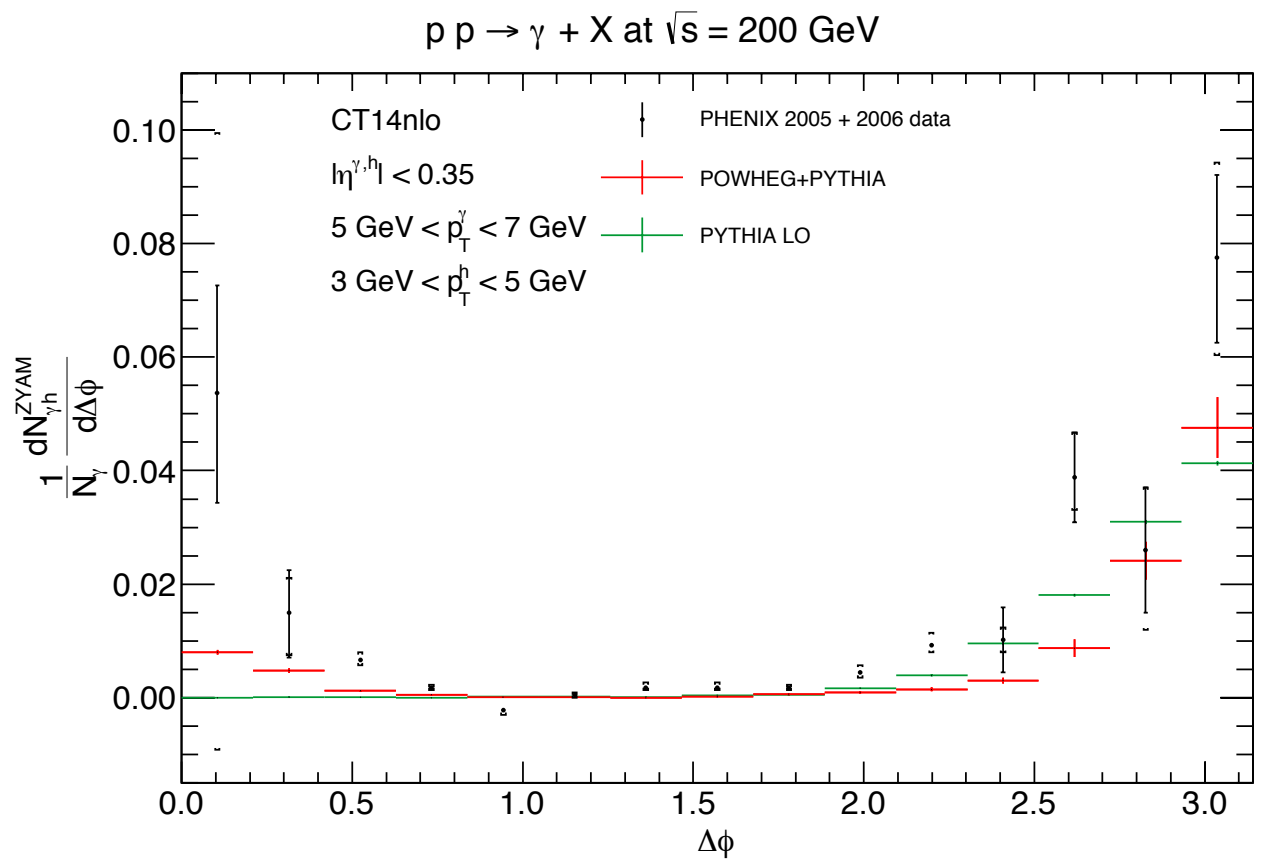

Figure 2: Azimuthal-angle correlation of the photon-hadron pair in pp collisions with $\sqrt{s}=200 \mathrm{GeV}$ at RHIC in LO+PS (green) and NLO+PS (red) and compared to PHENIX data (black) [7]. 


\section{Photon-jet correlations at the LHC}

Photon-jet correlations can also help us to improve on our knowledge of nuclear modifications of PDFs such as shadowing at low $x$. The reason is that Deep-Inleastic Scattering (DIS) and DrellYan (DY) processes constrain mostly quark and anti-quark nPDFs at intermediate and large $x$, respectively [8], while considerable uncertainties remain in the nuclear gluon and sea quark PDFs at small $x$. This could be changed by including in the global fits future LHC pPb data from photon production in association with jets (or heavy quarks) [9].

A useful variable to analyse photon-jet data directly in view of the nPDFs is the observed parton momentum fraction

$$
x_{\mathrm{Pb}}^{\mathrm{obs}}:=\frac{p_{T}^{\gamma} e^{-\eta^{\gamma}}+p_{T}^{\mathrm{jet}} e^{-\eta^{\mathrm{jet}}}}{2 E_{\mathrm{Pb}}}
$$

in the lead ion. As one can see, small values of $x$ are best accessed at small transverse momenta and/or forward rapidities. Fig. 3 shows the differential cross section of photon-jet production in $\mathrm{pPb}$

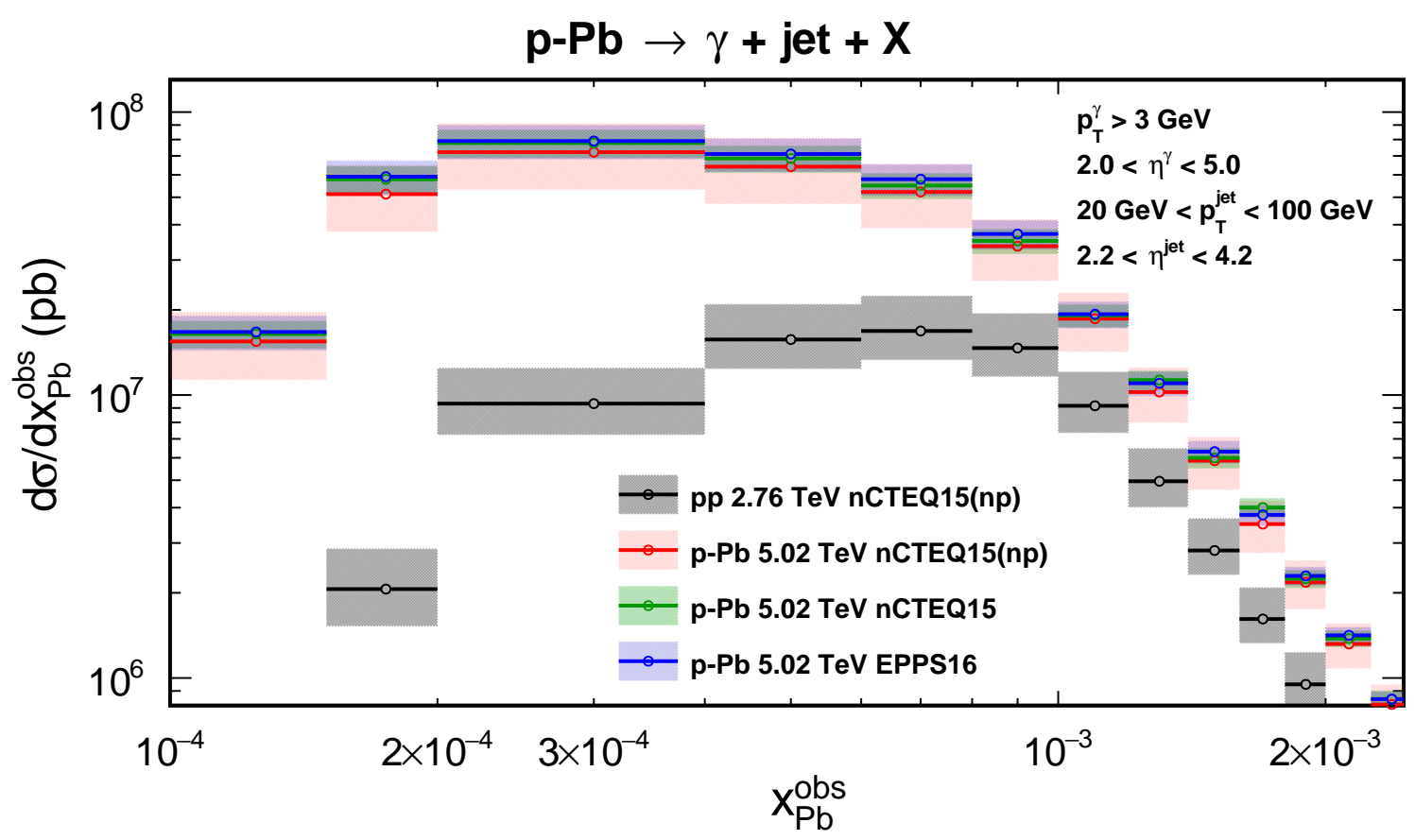

Figure 3: Differential distribution in the observed parton momentum fraction in lead ions in $\mathrm{pPb}$ collisions at the LHC [6].

collisions at the LHC with a centre-of-mass energy per nucleon of $\sqrt{s_{N N}}=5.02 \mathrm{TeV}$. As expected, data from DIS and DY alone (red) lead to large nPDF uncertainties at small $x$. The addition of pion production data from RHIC (green) and weak-boson and jet production data from LHC (blue) lead to similar improvements, which can also be expected from photon-jet data. For comparison, predictions for pp collisions at $2.76 \mathrm{TeV}$ including theoretical scale uncertainties are also shown (grey). 


\section{Conclusions}

In conclusion, we have computed photon-jet production at hadron colliders at NLO and matched this calculation to PS using the POWHEG method. This will allow in the future for predictions of photon and jet observables in $\mathrm{pp}, \mathrm{pPb}$ and $\mathrm{PbPb}$ collisions at RHIC or the LHC with reliable normalisation and realistic detail of the final state.

Choosing three typical observables, we have demonstrated this assertion for the transversemomentum balance and azimuthal-angle correlation at RHIC and for studies of nuclear PDF uncertainties at the LHC. The latter become easily accessible if the data are directly analysed in terms of the observed parton momentum fraction in the lead ion.

\section{Acknowledgments}

We thank T. Jezo, C. Klein-Bösing, F. König and H. Poppenborg for their collaboration and M. Cacciari, D. d'Enterria and Y.J. Lee for useful discussions.

\section{References}

[1] X. N. Wang, Z. Huang and I. Sarcevic, Phys. Rev. Lett. 77 (1996) 231; M. Klasen, C. Klein-Bösing, F. König and J. P. Wessels, JHEP 1310 (2013) 119.

[2] P. Skands, S. Carrazza and J. Rojo, Eur. Phys. J. C 74 (2014) 3024.

[3] S. Catani, M. Fontannaz, J. P. Guillet and E. Pilon, JHEP 0205 (2002) 028; P. Aurenche, M. Fontannaz, J. P. Guillet, E. Pilon and M. Werlen, Phys. Rev. D 73 (2006) 094007.

[4] S. Frixione, P. Nason and C. Oleari, JHEP 0711 (2007) 070.

[5] T. Jezo, M. Klasen and F. König, JHEP 1611 (2016) 033.

[6] M. Klasen, C. Klein-Bösing and H. Poppenborg, arXiv:1709.04154 [hep-ph].

[7] A. Adare et al. [PHENIX Collaboration], Phys. Rev. C 80 (2009) 024908.

[8] K. Kovarik et al., Phys. Rev. D 93 (2016) 085037; K. J. Eskola, P. Paakkinen, H. Paukkunen and C. A. Salgado, Eur. Phys. J. C 77 (2017) 163.

[9] T. Stavreva, I. Schienbein, F. Arleo, K. Kovarik, F. Olness, J. Y. Yu and J. F. Owens, JHEP 1101 (2011) 152; M. Brandt, M. Klasen and F. König, Nucl. Phys. A 927 (2014) 78; M. Klasen, K. Kovarik and J. Potthoff, Phys. Rev. D 95 (2017) 094013. 